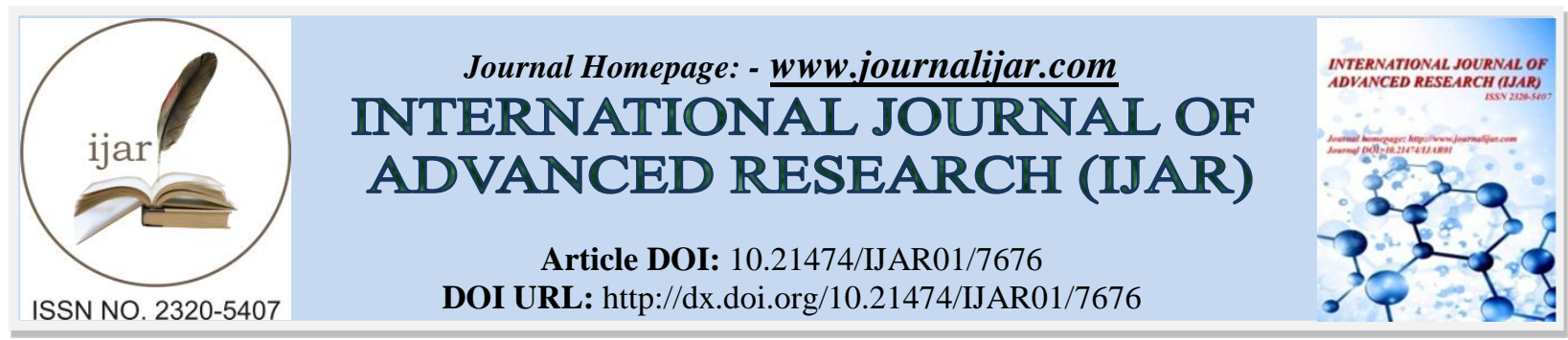

RESEARCH ARTICLE

\title{
EPISODIC MEMORY DEFICITS: COMPARISON BETWEEN PERSONS WITH DEMENTIA OF ALZHEIMER'S TYPE, MILD COGNITIVE IMPAIRMENT \& HEALTHY ELDERLY INDIVIDUALS.
}

Reuben Thomas Varghese ${ }^{1}$ and S.P. Goswami ${ }^{2}$.

1. Scientist B, Department of Speech- Language Sciences All India Institute of Speech \& Hearing, Mysuru, India.

2. Prof. in Speech Pathology, Department of Speech - Language Pathology All India Institute of Speech \& Hearing, Mysuru, India.

\section{Manuscript Info}

Manuscript History

Received: 06 July 2018

Final Accepted: 08 August 2018

Published: September 2018

Keywords:-

Episodic memory, Mild Cognitive Impairment, Dementia of Alzheimer's type.

\begin{abstract}
Episodic memory refers to the ability for recollecting an autobiographical memory of events that has occurred at a particular time and place. However, there is a dearth of episodic memory studies in Persons with Dementia of Alzheimer's type (DAT), Mild-Cognitive Impairment (MCI) and Healthy controls (HC). The aim of the present study was to compare episodic memory deficits across all the three groups. A total of sixty participants were recruited for the present study. These participants were categorized under three subgroups, twenty HC, twenty persons with MCI and twenty persons with Mild DAT. The participant groups were again sub divided into three agegroups [(>60-65yrs, $>65-70$ yrs \& $>70-75$ yrs $)]$. Episodic memory tasks were evaluated by using Orientation and recent memory test from the memory domain of Cognitive-Linguistic Assessment Protocol (CLAP, Kamath, 2001) to the participants. The results of the study revealed that persons with DAT exhibited more episodic memory deficits compared to MCI and HC suggesting cognitive impairments and word retrieval deficits. The present study throws light on cognitive markers which can help in the differential diagnosis among the three groups. Further research with more number of samples is warranted.
\end{abstract}

Copy Right, IJAR, 2018,. All rights reserved.

\section{Introduction:-}

Population aging has been a concern in India in the recent times. According to the Ministry of Statistics \& Program Implementation- Census, 2011 data, there are more than 104 million elderly in India. Cognitive -communication problems and dementia are the most common problems in the elderly population. Dementia describes an array of brain disorders which progressively lead to brain damage, and subsequently cause a gradual deterioration of a person's functional capacity and social relations. Cummings, Benson, and LoVerme (1980) reported that at least three of the following five domains - language, memory, visuo-spatial skills, emotion, personality and cognition must be disrupted for persons with dementia. Dementia of Alzheimer's Type (DAT) is one of the most common causes of dementia, affecting memory, language, thinking and behaviour of an individual in middle or older age groups. According to Diagnostic and Statistical Manual of Mental Disorders- Fourth Edition (DSM- IV), Mild Cognitive Impairment (MCI) refers to a transitional stage between cognitive stages of normal aging and mild dementia. Petersen et al. (2001) reported that individuals with MCI have an increased risk of progressing to

Corresponding Author:- Reuben Thomas Varghese.

Address:-. Scientist B, Department of Speech- Language Sciences All India Institute of Speech \& 300 
Alzheimer's Disease. Deficits in memory has received the most attention in aging population (Grady, 2008; Park \& Gutchess, 2002). Episodic memory refers to the ability for recollecting an autobiographical memory of events that occurred in a particular temporal and spatial context (Squire, 2004). The most important characteristic of episodic memory is autonoetic awareness. This helps an individual capable of movement at will in subjective time, by remembering past experiences, or imagining possible future experiences (Wheeler \& Buckner, 2004).Numerous studies have reported age-related changes in episodic memory in healthy individuals (Budson and Price, 2005; Kamath \& Prema, 2001), persons with MCI ( Irish et.al, 2001) and persons with Dementia (Sunil \& Shyamala 2009; Anjana \& Shanbal, 2010). The studies revealed that episodic memory scores deficits were more in dementia group and MCI group compared to the healthy elderly individuals. It was also noticed that memory deficits tend to increase with age. The temporal lobe, which comprises of the hippocampus, and the prefrontal cortex are key to episodic memory. Studies have reported that the hippocampus is one of the first brain structures damaged in both persons with Dementia of Alzheimer's type and Mild Cognitive Impairment (Zhan et al, 2016) compared to normal aging individuals. However, limited studies have been done in the Indian context comparing the episodic memory deficits in these populations. Hence, purpose of the present study was to compare the episodic memory deficits between Persons with Dementia of Alzheimer's type (DAT), Mild Cognitive Impairment (MCI) and normal healthy elderly controls (NHC) as well as across age groups.

The objectives of the study were:

1. To compare the episodic memory deficits in normal healthy elderly controls (HC) across age-groups $[(>60-$ $65 y r s,>65-70$ yrs \& $>70-75$ yrs)].

2. To compare the episodic memory deficits in persons with MCI across age-groups [(>60-65yrs, >65-70 yrs \& $>70-75$ yrs)].

3. To compare the episodic memory deficits in persons with DAT across age-groups $[(>60-65 \mathrm{yrs},>65-70$ yrs $\&$ $>70-75$ yrs)].

4. To compare the episodic memory deficits between persons with DAT, MCI \& NHC within age-groups [(>60$65 y r s,>65-70$ yrs \& $>70-75$ yrs $)]$.

\section{Method:-}

The research design taken for the present study was standard group comparison. A total of sixty participants were recruited for the present study. These participants were categorized under three subgroups, namely group I, group II and group III. The participants included in group I were twenty normal healthy controls (NHC), participants in group II were twenty persons with Mild Cognitive Impairment (MCI) and participants in group III were twenty persons with Mild Dementia of Alzheimer's type (DAT). All the three participant groups were again sub divided into three age-groups $[(>60-65 y r s,>65-70$ yrs $\&>70-75$ yrs $)]$. The participants in group II and group III were identified through local hospitals, dementia support groups and dementia clinics in Mysuru city, Karnataka, India The selection criteria common for all the groups were as follows:

1. They were above the age of 60 years

2. They were native Kannada speakers with adequate hearing and vision (corrected). For hearing screening, Lings six sound test and for vision Zeiss Online vision Screening Test was considered.

3. The opinion of the neurologist/ psychiatrist was considered for participant selection.

4. All participants were evaluated for their mental status on Mini Mental State Examination (Folstein, Folstein \& McHugh, 1975) and those who failed below a score of 23 were taken for group II and group III and participants who had passed a score of 23 and above (indicates no cognitive impairment) were taken for the Group I.

\section{Selection Criteria specific for Group I:-}

Participants with no history of hoarse voice, major neurological or psychiatric illness or of alcoholism or drug abuse were included.

\section{Selection criteria specific for Group II:-}

The opinion of the neurologist/ psychiatrist according to MoCA-Montreal Cognitive Assessment Scale (Nasreddine, Bédirian, Charbonneau, Whitehead, Collin, Cummings \& Chertkow, 2005) and Peterson criteria was considered for selecting MCI group.

\section{Selection criteria specific for Group III:-}

The opinion of the neurologist/ psychiatrist according to NINCDS- ADRDA (National Institute of Neurological Disorders- Alzheimer's Disease and Related Disorders Association, 2007) was taken for selecting DAT group. 
The study was carried out after the approval of the AIISH ethical committee and adhered to the ethical guidelines stated by AIISH, Mysore. Karnataka, India. Informed consent was taken from the participant / caregiver for the recording.

\section{Material:-}

Cognitive-Linguistic Assessment Protocol (CLAP, Kamath, 2001) is a test which assesses the cognitive and linguistic abilities in both young and elderly population. This test assess attention, perception \& discrimination, memory, problem solving and organization.

\section{Procedure}

Each participant was seated in a noise free room. Episodic memory tasks were evaluated by using Orientation and recent memory test from the memory domain of Cognitive-Linguistic Assessment Protocol to the participants. The task was evaluated by asking questions to the participant with respect to orientation like place, time, self and a few questions pertaining to general knowledge and recent memory. The participant had to answer a total of 10 questions and 1 mark was given for each correct answer. The maximum total score for the test was 10.

\section{Statistical Analysis}

The raw scores were tabulated and subjected to statistical analysis. Shapiro Wilks test of normality was carried out. As the data was not following assumptions of normality at 0.05 level of significance, non parametric test KruskalWallis test was carried out to see the significant difference between participant groups and age groups. Further, Mann Whitney test was carried out to find pair-wise significant difference between age-groups and participant groups (HC, MCI \& DAT). These analyses were carried using SPSS 21 version.

\section{Results:-}

The aim of the present study was to compare the episodic memory deficits across three age groups- group I ( $>60$ 65 yrs), group II (>65-70yrs) and group III (>70-75yrs) between Persons with Dementia of Alzheimer's type (DAT), Mild Cognitive Impairment (MCI) and normal healthy controls (HC) using Cognitive-Linguistic Assessment Protocol (CLAP). Episodic memory tasks were evaluated by using orientation and recent memory test from the memory domain of Cognitive-Linguistic Assessment Protocol to the participants. The task was evaluated by asking questions to the participant with respect to orientation like place, time, self and a few questions pertaining to general knowledge and recent memory. The participant had to answer a total of 10 questions and 1 mark was given for each correct answer. The maximum total score for the test was 10 .

\section{Episodic memory deficits in NHC across age- groups}

It was noticed that the mean score for NHC for episodic memory task for age group I was 9.91, age group II was 9.82 and age group III was 9.41. The overall mean score for all the age groups were 9.76. Kruskal - Wallis test revealed that there is a significant difference among different age groups for healthy controls $\left(\chi^{2}(2)=9.24\right.$, $p<0.001)$.Further, Mann Whitney test revealed that there no significant difference between age group I \& age group II $[|z|=0.874 p>0.01$ ], but there was significant difference between age group II \& age group III [ $|z|=2.154, p<0.01]$ and age group I and age group III $[|z|=2.870, p<0.01]$ in HC group. Table I depicts the Mean, Std.Deviation, Median \& Mean Rank for episodic memory scores in NHC across age- groups.

Table I:-Mean, Standard deviation (SD), Median and Mean Rank for Episodic memory scores in NHC across agegroups.

\begin{tabular}{|l|c|c|c|c|c|c|}
\hline Group & Age group & $\mathrm{N}$ & Mean & Std. Deviation & Median & Mean Rank \\
\hline \multirow{4}{*}{ NHC } & $>60-65$ & 10 & 9.91 & .307 & 10.00 & 29.00 \\
\cline { 2 - 7 } & $>65-70$ & 5 & 9.82 & .410 & 10.00 & 26.51 \\
\cline { 2 - 7 } & $>70-75$ & 5 & 9.41 & .517 & 9.00 & 16.50 \\
\cline { 2 - 7 } & Total & 20 & 9.76 & .431 & 10.00 & \\
\hline
\end{tabular}

\section{Episodic memory deficits in MCI across age- groups}

It was noticed that the mean score for persons with MCI for episodic memory task for age group I was 9.0, age group II was 8.42 and age group III was 7.64. The overall mean score for all the age groups were 8.48. Kruskal - Wallis test revealed that there is a significant difference among different age groups for MCI group $\left(\chi^{2}(2)\right.$ $=15.612, p<0.001)$. Further, Mann Whitney test revealed that there was significant difference between age 
group I \& age group II $[|z|=2.8754, p<0.05]$, age group II \& age group III $[|z|=2.239, p<0.01]$ and age group I and age group III $[|z|=3.669, p<0.001]$ in MCI group. Table II depicts the Mean, Std.Deviation, Median \& Mean Rank for episodic memory scores in persons with MCI across age- groups.

Table II:-Mean, Standard deviation (SD), Median and Mean Rank for Episodic memory scores in MCI across agegroups.

\begin{tabular}{|l|c|c|c|c|c|c|}
\hline Group & Age group & $\mathrm{N}$ & Mean & Std. Deviation & Median & Mean Rank \\
\hline \multirow{4}{*}{ MCI } & $>60-65$ & 10 & 9.00 & 0.00 & 9.00 & 18.51 \\
\cline { 2 - 7 } & $>65-70$ & 5 & 8.42 & .516 & 8.00 & 11.61 \\
\cline { 2 - 7 } & $>70-75$ & 5 & 7.64 & .549 & 8.00 & 4.90 \\
\cline { 2 - 7 } & Total & 20 & 8.48 & .653 & 9.00 & \\
\hline
\end{tabular}

Episodic memory deficits in DAT across age - groups

It was noticed that the mean score for persons with DAT for episodic memory task for age group I was 7.31, age group II was 6.20 and age group III was 4.22. The overall mean score for all the age groups were 6.24. Kruskal Wallis test revealed that there is a significant difference among different age groups for healthy controls $\left(\chi^{2}(2)\right.$ $=9.24, p<0.05)$.Further, Mann Whitney test revealed that there was significant difference between age group I $\&$ age group II $[|z|=3.245 p<0.05]$, age group II \& age group III $[|z|=2.239, p<0.05]$ and age group I and age group III $[|z|=2.870, p<0.05]$ in DAT group. Table III depicts the Mean, Std.Deviation, Median \& Mean Rank for episodic memory scores in DAT across age- groups.

Table III:-Mean, Standard deviation (SD), Median and Mean Rank for Episodic memory scores in DAT across age- groups.

\begin{tabular}{|c|c|c|c|c|c|c|}
\hline Group & Age group & $\mathrm{N}$ & Mean & Std. Deviation & Median & Mean Rank \\
\hline \multirow{4}{*}{ DAT } & $>60-65$ & 10 & 7.31 & .482 & 10 & 19.42 \\
\cline { 2 - 7 } & $>65-70$ & 5 & 6.20 & .652 & 10 & 11.51 \\
\cline { 2 - 7 } & $>70-75$ & 5 & 4.22 & .441 & 5 & 3.10 \\
\cline { 2 - 7 } & Total & 20 & 6.24 & 1.268 & 25 & \\
\hline
\end{tabular}

Episodic memory deficits between NHC,MCI \& DAT across age- groups

Kruskal Wallis test was used to find significant difference among participant groups across different age groups. The results revealed that there was a significant difference in episodic memory between NHC, MCI \& DAT for agegroup I $\left[\left(\chi^{2}(2)=35.181, p<0.001\right)\right]$, age-group II $\left[\left(\chi^{2}(2)=33.906, p<0.001\right)\right]$ and age-group II $\left[\left(\chi^{2}(2)=16.831\right.\right.$, $p<0.001)]$.

\section{Comparison between NHC and MCI}

The results revealed that there was significant difference between NHC and MCI in age-group I $[|z|=4.664$, $p<0.001]$, age-group II $[|z|=4.664, p<0.001]$ and age-group III $[|z|=3.208, p<0.05]$.

\section{Comparison between MCI and DAT}

The results revealed that there was significant difference between NHC and MCI in age-group I $[|z|=4.147$, $p<0.001]$, age-group II $[|z|=3.905, p<0.001]$ and age-group III $[|z|=2.739, p<0.05]$.

\section{Comparison between NHC and DAT}

The results revealed that there was significant difference between NHC and DAT in age-group I $[|z|=5.011$, $p<0.001]$, age-group II $[|z|=4.806, p<0.001]$ and age-group III $[|z|=3.224, p<0.05]$.

Table IV:-Mean, Standard deviation (SD), Median for Episodic memory scores for NHC,MCI \& DAT across agegroups.

\begin{tabular}{|c|c|c|c|c|c|c|c|c|c|c|c|c|}
\hline & \multicolumn{4}{|c|}{ NORMAL } & \multicolumn{4}{|c|}{ MCI } & \multicolumn{4}{|c|}{ DAT } \\
\hline $\begin{array}{c}\text { AGE - } \\
\text { GROUP }\end{array}$ & $\mathrm{N}$ & MEAN & $\mathrm{SD}$ & MDN & $\mathrm{N}$ & MEAN & SD & MDN & $\mathrm{N}$ & MEAN & $\mathrm{SD}$ & MDN \\
\hline$>60-65$ & 10 & 9.91 & .307 & 10.00 & 10 & 9.00 & 0.000 & 9.00 & 10 & 7.31 & .482 & 7.00 \\
\hline$>65-70$ & 5 & 9.82 & .410 & 10.00 & 5 & 8.42 & .516 & 8.00 & 5 & 6.20 & .652 & 6.00 \\
\hline
\end{tabular}




\begin{tabular}{|l|l|l|l|l|l|l|l|l|l|l|l|l|}
\hline$>70-75$ & 5 & 9.41 & .517 & 9.00 & 5 & 7.64 & .549 & 8.00 & 5 & 4.22 & .441 & 4.00 \\
\hline
\end{tabular}

Thus from table IV, we have the complete summary of mean scores in episodic memory tasks between Normal, Mild DAT \& At Risk for DAT individuals .Based on the above scores, it can help clinicians and researchers to identify early markers in episodic memory to identify based on the scores and classify them according to normal aging, MCI or DAT.

\section{Discussion:-}

The study was undertaken to compare the episodic memory deficits across three age ranges (60-65yrs, 65-70yrs \& 70-75yrs) between normal healthy controls (NHC), Persons with Mild Cognitive Impairment(MCI) \& Persons with Dementia of Alzheimer's type (DAT) and using semantic fluency tasks. The discussion of the study is presented in the following subheadings

\section{For Healthy controls}

From the results, it was evident that the episodic memory scores decreased from age group I ( $>60$-65), age group II $(>65-70)$ and age-group III $((>70-75)$. The decrease in the scores indicates more memory deficits. However the difference in mean scores was minimal, 0.09 for age group I and age group II, 0.41 for age-group II and age group III and 0.50 for age-group I and age-group III. This suggests that that episodic memory plays an important role in the ability to search and retrieve information. The above results are in consensus with the previous studies which show age related decline in the cognitive skills of older individuals (Budson and Price, 2005; Kamath \& Prema, 2001). Thus, more errors while answering questions related to place, time etc be associated to the age related episodic memory deficits in normal healthy controls.

\section{For Mild Cognitive Impairment}

From the results, it was evident that the episodic memory scores decreased from age group I (>60-65), age group II $(>65-70)$ and age-group III ((>70-75). However the difference in mean scores was 0.58 for age group I and age group II, 0.78 for age-group II and age group III and 1.36 for age-group I and age-group III. The decrease in the scores indicates more memory deficits. Studies have reported that persons with MCI progress to DAT in the later years and episodic memory deficits are one of the hallmarks seen in persons with MCI (Irish et.al, 2001, Zhan, 2016).

\section{For Persons with DAT}

From the results, it was evident that the episodic memory scores decreased from age group I (>60-65), age group II $(>65-70)$ and age-group III ((>70-75). However the difference in mean scores was 1.1 for age group I and age group II, 1.98 for age-group II and age group III and 3.09 for age-group I and age-group III. The decrease in the scores indicates more memory deficits. Studies have reported episodic memory deficits in DAT (Sunil \& Shyamala 2009; Anjana \& Shanbal, 2010).

\section{Comparison between the groups}

Since the standard deviation is very small, the mean scores was used as it provided a good representation. Thus from the results, we can infer that for age group I (>60-65yrs), the difference in mean scores between NHC and MCI was 0.9.The difference in mean scores between MCI and DAT was 1.69. The difference in mean scores between NHC and DAT was 2.60. For age group II (>65-70), the difference in mean scores between NHC and MCI was 1.4. The difference in mean scores between MCI and DAT was 2.22. The difference in mean scores between NHC and DAT was 3.62. For age group III (>70-75), the difference in mean scores between NHC and MCI was 1.77.The difference in mean scores between MCI and DAT was 3.42. The difference in mean scores between NHC and DAT was 5.19.Thus we can infer that as age increased, the episodic memory deficits between each participant group also increased, being more prominent for the DAT group. The decrease in the episodic memory might be due to the reduction in hippocampal and entorhinal cortex volumes in groups which might have also been affected by age advancement.

\section{Summary \& Conclusion:-}

The above study was an attempt to investigate the episodic memory deficits between normal healthy controls (NHC), Persons with Mild Cognitive Impairment (MCI) and Persons with Dementia of Alzheimer's type (DAT) using memory domain of Cognitive-Linguistic Assessment Protocol (CLAP). The results of the study revealed that 
persons with DAT exhibited more episodic memory deficits compared to MCI \& NHC . This study also throws also light on early cognitive markers which can help in the differential diagnosis among all the groups. The above study also gives out red flags in terms of cognitive measures for MCI individuals which can help clinicians to plan out strategies to prevent cognitive slowing. Further research with more number of samples is warranted.

\section{Appendix}

\section{Orientation and Recent memory questions}

The questions will be as follows

1. nimma hesaru eenu?

2. nimma taii hesaru eenuira?

3. Niivu uTa yavaga maDuttira? BeLage/maDhyana/ sanje/ ratri?

4. Idu yavu uuru?

5. bharatada iigna pradhanamantry yaru?

6. Sauuryannu puurvadalli hu Tuttano pascimadallo?

7. tandeya tandege yenu andu hesaru?

8. Banknalli Ittiruva haNakke prati tingal baruva sampadaneege- endu hesaru

9. Ondu padada arthavannu niivu yelli huDukalu?

10. March tingLu, june tingLina munce baratta?

Scoring: 1 mark will be given for every correct answer

Total Score: Correct out of 10

\section{Acknowledgement:-}

The authors would like to thank Dr. S.R. Savithri, Director AIISH, for permitting us to carry out the study. The authors would also like to thank the participants for their support and co-operation.

\section{Conflict of Interest:-Nil}

\section{References:-}

1. Anjana, A. V., \& Shanbal, J .(2010): Cognitive Communication Assessment Protocol for Persons with Dementia - Malayalam. Students Research at All India Institute of Speech and Hearing, Part B (VIII):12-28.

2. American Psychiatric Association, (1994): Diagnostic and statistical manual of mental disorders. Washington, Am Psychiatr Assoc, pp.143-146.

3. Budson, A.E. and Price, B.H., (2005): Memory dysfunction. New England Journal of Medicine, 352(7):692699.

4. Chandramouli, C. and General, R.( 2011): Census of India 2011. Provisional Population Totals. New Delhi: Government of India.

5. Cummings, J., Benson, D.F. and LoVerme, S. (1980): Reversible dementia: illustrative cases, definition, and review. Jama, 243(23):2434-2439.

6. Folstein, M.F., Folstein, S.E. and McHugh, P.R.(1975): "Mini-mental state": a practical method for grading the cognitive state of patients for the clinician. Journal of psychiatric research, 12(3):189-198.

7. Grady, C.L.( 2008): Cognitive neuroscience of aging. Annals of the new york Academy of Sciences, 1124(1):127-144.

8. Irish, M., Lawlor, B.A., O’Mara, S.M. and Coen, R.F.(2010): Exploring the recollective experience during autobiographical memory retrieval in amnestic mild cognitive impairment. Journal of the International Neuropsychological Society, 16(3):546-555.

9. Kamath, A., \& Prema, K. S. (2001): Cognitive - Linguistic Assessment Protocol for adults. Research at AIISH, Dissertation abstracts: Vol (IV): 124-125.

10. Nasreddine, Z.S., Phillips, N.A., Bédirian, V., Charbonneau, S., Whitehead, V., Collin, I., Cummings, J.L. and Chertkow, H. (2005): The Montreal Cognitive Assessment, MoCA: a brief screening tool for mild cognitive impairment. Journal of the American Geriatrics Society, 53(4):695-699.

11. Park, D. and Gutchess, A. (2006): The cognitive neuroscience of aging and culture. Current directions in psychological science, 15(3):105-108. 
12. Petersen, R.C., Doody, R., Kurz, A., Mohs, R.C., Morris, J.C., Rabins, P.V., Ritchie, K., Rossor, M., Thal, L. and Winblad, B..(2001). Current concepts in mild cognitive impairment. Archives of neurology, 58(12): 19851992.

13. Sunil, K. R., \& Shyamala, K. C. (2009): Dementia Assessment Battery in Kannada. Unpublished masters' dissertation submitted to University of Mysore. Mysore.

14. Squire, L.R. (2004): Memory systems of the brain: a brief history and current perspective. Neurobiology of learning and memory, 82(3):171-177.

15. Wheeler, M.E. and Buckner, R.L.( 2004): Functional-anatomic correlates of remembering and knowing. Neuroimage, 21(4):1337-1349.

16. Zhan, Y., Ma, J., Alexander-Bloch, A.F., Xu, K., Cui, Y., Feng, Q., Jiang, T. and Liu, Y.(2016) :Longitudinal study of impaired intra-and inter-network brain connectivity in subjects at high risk for Alzheimer's disease. Journal of Alzheimer's Disease, 52(3):913-927. 